\title{
Gaussian ISI Channels and the Generalized Likelihood Ratio Test
}

\author{
Amos Lapidoth \\ ETF E 107 ETHZ \\ CH-8092 Zürich \\ Switzerland \\ lapidoth@isi.ee.ethz.ch
}

Abstract - Decoders employing the generalized likelihood ratio test can achieve rates that can be achieved by maximum likelihood decoders on ISI channels even though they are ignorant of the channel characteristics.

\section{INTRODUCTION}

A variety of communication systems can be modeled accurately by an intersymbol interference (ISI) channel. In many situations, however, the exact nature of the interference may not be known at the time of the system design. In this note, we consider the performance of a particular decoding rule that, in contrast to maximum likelihood (ML), operates with imprecise knowledge of the channel.

The question of the existence of universal decoders for the ISI channel has been previously addressed [1]. It is known that universal decoders do exist for this class of channels. However, the existence proof suggests a very complicated construction, one that requires to consider all ML decoders for all the possible ISI channels, and to form a "merging" of these decoders into a single universal one. As such, the complexity of the evaluation of any particular codeword is very high.

For the case of discrete memoryless channels the situation is simpler. The maximum mutual information decoder first suggested in [2] and widely popularized in [3] employs a relatively simple decoding rule: given a received sequence $\mathbf{y}$, and a candidate codeword $\mathbf{x}$, compute a score $\max _{Q} Q(\mathbf{y} \mid \mathbf{x})$, where the maximization is taken over all DMC probability laws. The decoder then chooses the codeword with the highest score. It is known that this decoding rule is universal. Even though the cost of codeword evaluation is more than that of maximum likelihood decoding, it is still much less than that of universal decoders based on merging.

The natural generalization of the above decoding rule leads to the so called "Generalized Likelihood Ratio Test" (GLRT): Let the possible channels be parametrized by $\theta$ with $P_{\theta}$ denoting the probability law of the corresponding channel. Given a received sequence $\mathbf{y}$, compute the score of $\mathbf{x}$ as $\max _{\theta} P_{\theta}(\mathbf{y} \mid \mathbf{x})$ and choose the codeword with the highest score.

That universal decoders do exist for the ISI channel does not imply that the GLRT decoder performs well; there are classes of channels for which there exists a universal decoder, but GLRT performs poorly [4]. In this presentation, we will investigate the performance of the GLRT on ISI channels. In particular we will show that as far as achievable rates are concerned, the GLRT decoder performs as well as the maximum likelihood decoder.

\section{RESULTS}

If the spectral characteristics of an ISI channel are known in advance, the codebook used over this channel will be designed accordingly; in particular, the capacity of the channel can be achieved via water pouring. Since we assume that the ISI coefficients are not known in advance, we will consider the case

\author{
Emre Telatar \\ EPFL - DSC - LTHI \\ $\mathrm{CH}-1015$ Lausanne \\ Switzerland \\ Emre.Telatar@epfl.ch
}

in which the codewords are chosen to have a flat spectrum. We will content ourselves by considering the rates achievable by GLRT decoders and ML decoders when the codebook is chosen as such. Since the codebook is not spectrally matched to the channel we have no hope of achieving the true capacity of the channel; but we feel that to have assumed that the transmitter is designed with the knowledge of the channel whereas the receiver is not would have been artificial. In all the cases considered below, the transmitter is subject to an average power constraint $P$.

We will assume that the channel filter $\alpha$ has at most a given duration $J$, and that the output of the channel at time $k$ is related to the channel input $\mathbf{x}$ via

$$
Y_{k}=(\boldsymbol{\alpha} * \mathbf{x})_{k}+Z_{k}, \quad k=1, \ldots, n,
$$

where $*$ denotes cyclic convolution and $Z_{k}$. are i.i.d, circularly symmetric Gaussian random variables with $E\left[Z_{1}\right]=0$, $E\left[\left|Z_{1}\right|^{2}\right]=1$. The use of cyclic convolutions is motivated for reasons of analytical convenience, but can be justified by prepending each codeword by its last $J$ symbols.

In addition to assuming that $\alpha_{k}=0$ for $k \geq J$, we will further assume that the filter $\alpha$ satisfies a norm constraint $\sum_{j}\left|\alpha_{j}\right|^{2} \leq H$.

The GLRT decoder then works as follows: given a received $\mathbf{y}$, it assigns to each codeword $\mathbf{x}$ a score $\min _{\mathbf{a}}\|\mathbf{y}-\mathbf{a} * \mathbf{x}\|$ where the minimum is taken over all filters a of at most $J$ taps that satisfy the energy constraint $\|\mathbf{a}\|^{2} \leq H$. The decoder then declares the codeword of smallest score.

We show that for randomly chosen codes (with independently chosen codewords, each codeword chosen either uniformly on the sphere or with i.i.d. Gaussian components), the error probability for the GLRT decoder decays to zero as long as the code rate is less than

$$
\int_{0}^{1} \log \left(1+P|\alpha(\theta)|^{2}\right) d \theta
$$

where $\alpha(\theta)=\sum_{k} \alpha_{k} e^{i 2 \pi \theta k}$ is the Fourier transform of the channel impulse response. We thus see that for ISI channels, GLRT decoders can achieve all rates the ML decoder can.

\section{REFERENCES}

[1]. M. Feder and A. Lapidoth, "Universal Decoding for Channels with Memory," IEEE Transactions on Information Theory, v. 44 , no. 5, pp. 1726-1745, September, 1998.

[2] V. D. Goppa, "Nonprobabilistic mutual information without memory," Problems in Controls and Information Theory, v. 4, pp. 97-102, 1975.

[3] I. Csiszar and J. Körner, Information Theory: Coding Theorems for Discrete Memoryless Systems, New York: Academic Press, 1981.

[4] A. Lapidoth and J. Ziv, "On the Universality of LZ-Based Decoding Algorithm," IEEE Transactions on Information Theory, v. 44, no. 5, pp. 1746-1755, September, 1998. 